\title{
Eye-tracking for efficient database labelling: Applications to automatic analysis of colonoscopy video
}

\author{
Fernando Vilarino and Gerard Lacey \\ Graphics, Vision and Visualisation Group (GV2) \\ Computer Science Department, Trinity College Dublin (TCD), Rep. of Ireland \\ fernando.vilarino@cs.tcd.ie
}

\begin{abstract}
In this paper we present our preliminary results in the automatic analysis of colonoscopy video using eye-tracking. We propose that eye-tracking can be successfully applied to solve different problems in computer assisted colonoscopy, such as database labelling, expertise assessment and abnormality detection. We provide results in these three areas, including a machine learning-based system for colon cancer detection using data generated with eye-tracking.
\end{abstract}

\section{Introduction}

Colonoscopy is the ultimate technique for the diagnosis of several abnormalities in the colon tissue, including colon cancer. Still, different works have reported miss rates over $20 \%$ for certain types of colon cancer [2]. In this scenario, automatic systems based on colonoscopy video analysis can help the experts by providing support tools for the clinical environment. However, one of the main difficulties that computer engineers find is the lack of massive databases to be used in the different training stages. We propose the use of eye-tracking for an efficient database build-up.

\section{Automatic colonoscopy video analysis}

Eye-tracking consists of the tracing of eyes during a screening session to determine the region of the video to which the expert is paying attention. We successfully used eye-tracking for the labelling of a video database, and applied it in three different applications: 1) Comparison of the visual trajectories for expertise skills assessment, 2) Development of a cancer vs. non-cancer database for successfully training a system which implements automatic cancer detection using a support vector machine (SVM) classifier [3], and 3) Characterisation of the saliency models associated with the different types of cancer.

\section{Results}

Our data consisted of 20 videos of about $45 \mathrm{sec}$. long containing both cancer and healthy tissue. We asked 3 experts and 3 novices, who carried an eye-tracking device, to screen all the videos. For the expertise assessment we plotted the line trajectories described by the eye position along the time in each video, and therefore we analysed the differences between experts and novices. For the development of the cancer vs. non-cancer detection, we asked the experts to select those frames in which cancer was visible. The eye-tracked region containing cancer was automatically segmented and stored. An equivalent number of non-cancer regions was randomly selected; a global number of 11,125 cancer and non-cancer images was obtained. All these raw images were used to train a SVM classifier which was tested in a leave-one-out strategy, obtaining $80 \%$ sensitivity and $20 \%$ of false positives rate. For the saliency identification, we clustered the obtained images with a self organising map (SOM) [1]. Each cluster was inspected and characterisation was performed regarding the types of polyps.

\section{Conclusions}

Eye-tracking arises as a promising technique for massive video database labelling, suitable of being used for automatic computer vision-based systems. Automatic colon cancer detection appears as a direct application for this novel methodology, showing good preliminary results.

\section{References}

[1] T. Kohonen. Self-Organized Maps. Springer, Heidelberg Berlin, 1995.

[2] D. Rex, C. Cutler, et al. Colonoscopic miss rates of adenomas determined by back-to-back colonoscopies. Gastroenterology, 112(1):292-4, 1997.

[3] V. Vapnik. The Nature of Statistical Learning Theory. Springer-Verlag, 1995. 